\title{
An Intelligent System to Detect the Type of Devices Sending and Receiving Data in the Network
}

\author{
Diana Bri ${ }^{1}$, Alejandro Canovas ${ }^{2}$, Jesus Tomas ${ }^{3}$ and Jaime Lloret ${ }^{4}$ \\ Integrated Management Coastal Research Institute, Polytechnic University of Valencia \\ C/ Paranimf, 1, Gandia, Valencia (Spain) \\ E-mail: 1diabrmo@upv.es, ${ }^{2}$ alcasol@upv.es, ${ }^{3}$ jtomas@upv.es, ${ }^{4}$ jlloret@upv.es
}

Received: May 25, 2013

Accepted: June 19, 2013

Published: June 30, 2013

DOI: 10.5296/npa.v5i2.3833

URL: http://dx.doi.org/10.5296/npa.v5i2.3833

\begin{abstract}
Nowadays mobile and fixed devices are used interchangeably for surfing the web due to the huge improvements performed in mobile devices in the recent years. Both mobile and fixed devices with Internet connectivity are supplied with different types of connection, thus users can select the best one at any time depending on their environment. In general, the mobile devices allow users access to Internet using the $3 \mathrm{G}$ network or a common WiFi connection, and the fixed ones generally use a wireless or wired connection. Selecting one or another type of connection implies different features of the network environment, so Internet Service Providers need to adapt their infrastructure to guarantee acceptable levels of Quality of Service in every type of connection. In this paper we study the behavior of the devices according to their nature, that is, if it is a mobile or fixed device. First, we have classified the most significant network parameters and software application values in order to know the nature of the device. Our proposal uses an intelligent system based on neural networks and finite state machines that lets the Internet Service Provider know the type of device belongs to the traffic going to its network. The system analyzes the transport and application layers from TCP packets to discriminate the percentage of Internet traffic generated by mobile and fixed devices. Test results show the success of the developed system.
\end{abstract}

Keywords: Device detection, Network Protocols, Traffic classification, Traffic Engineering. 


\section{Introduction}

Internet traffic generated by mobile devices has grown impressively in recent years. They can access to Internet using $3 \mathrm{G}$ or any other technology such as WiFi. It is important for mobile phone providers and Internet Service Providers to know which device is using their infrastructure in order to accommodate it to the user's demand or even study the most appropriate offers [1].

Traffic classification mechanisms allow allocating resources according to the service provider rules in TCP/IP networks. Moreover, it lets providers control and manage these resources, and enhance the security by knowing the type of traffic or device accessing Internet. In addition, it will help to deploy QoS-aware mechanisms successfully.

Most papers seek different techniques to classify IP traffic [2,3,4] and several methods have been researched. First of all, the port-based classification [5] was considered in order to identify the application that generated each flow by its transport level source and destination ports, but today this method has been rejected because well-known port numbers cannot be assumed to indicate the application reliably [6]. Then, other methods have been studied, such as statistical classification in order to probabilistically assign flows to classes, e.g., machine learning [2] or statistical clustering [3,4,6,7], payload-classification [8] or multi-level methodology [9].

But, our goal is to identify the type of device connected to Internet because we consider that, by knowing this information, it is easier to predict the behavior of users on the network, or foresee the connection time or level of attention to the requested service [10], regardless of the type of traffic which is generating. Moreover, providers will be able to offer better services for users adapting the transmission to the end-device because usually mobile devices have fewer resources than fixed ones.

The first problem that must be solved is to know if it is possible to recognize the operating system of the host connected behind a router by monitoring small variations in certain variables. Some recent studies have proved that some type of traffic classification can be performed $[11,12]$. Moreover, traffic variations generate patterns based on the operating system, which can be recognized by an intelligent system. The main issue is to determine the appropriate variables to be included in the intelligent system.

The objective of this work is to develop a TCP/IP classifier that is capable to distinguish between mobile devices (phones, smartphones, tablets, etc...) and fixed devices (running Windows, Linux or Mac OS). In order to achieve our goal, we will use statistical techniques applied to artificial intelligence. This paper is an extension of the paper published in [13].

The remainder of this paper is structured as follows. Section 2 shows the main related works. Section 3 explains the experiments performed to know the variables that can be used in the intelligent system. Our device recognition system is introduced in Section 4. Section 5 describes the experimental framework used to validate our approach. Finally, section 6 shows the conclusion and future work. 


\section{Related Works}

Device fingerprinting identification can be classified into two groups: active and passive approach.

In active approach the system send specific requests to the device and analyzes the response to detect implementation-specific characteristics. D. E. Comer and J. C. Lin demonstrated in [11] that active probing can be used to recognize TCP implementations. Therefore, this technique is only applicable to devices acting as server.

In the passive approach, the system only analyzes the traffic sent by devices. In [12], authors developed a naive Bayesian classifier based on TCP/IP fingerprints. This classifier is able to count the number of host devices masquering behind a Network Address Translation (NAT) router, analyzing the network messages, based on four variables: time to live (TTL), don't fragment bit (DF), window size (WS) and options block size (OBS).

Some statistical techniques applied to artificial intelligence have been implemented in several works with success. An example is the work presented by E. Hjelmvik and W. John in [14]. They introduce a statistical protocol identification algorithm using various statistical flow and application layer data features.

Most published studies focus on detecting some characteristic parameters of the software implementation, such as the browser type or the TCP/IP stack. In [15], the author introduces the HTTP fingerprinting technique to identify the server operating system. In [16], the author demonstrates that browser fingerprinting is a powerful technique that reveals quite a lot of information that remains overwhelmingly tractable with the implementation of a new browser fingerprinting method. Moreover, J. Oshio et al. [17] propose a new clustering method for controlling the congestion window, identifying different versions of TCP at the relay router in real time.

It is very important for Internet Service Providers to know which types of devices are using their infrastructure in order to provide the best quality of experience to the end user. In this line we propose a method to identify whether the traffic has been generated by a mobile device or by a fixed device. Our system uses a passive approach to capture the network traffic and artificial intelligence techniques to classify the Internet packets into two classes: mobile device and fixed device. But, as far as we know there is not any device fingerprint detection system like the one described in this paper.

\section{Test bench Measurements}

In order to see where we can find the information that can be used for our purpose, we performed several experiments. The topology used in these experiments is shown in Fg 1 . We placed all devices in a local area network using a single public IP address for Internet access. The local area network has 2 networks separated by a router. One of them uses Network Address Translation and is used to place all devices to be monitored: a PC with Windows XP, and a PC with Ubuntu 10.04, and a WiFi access point to connect the wireless devices: Nokia 


\section{Macrothink

C7-00 with Symbian OS 9.5 Symbian^3, iPhone with OS 6_1_2, SonyEricsson with Android 2.3.7, Nokia C6 SymbOS with S60, LG-p700 with Android 4.0.3, a tablet Acer with Android 3.2.1 and a MacBook Pro with Mac OSX 10.6.8. They use private IP addresses. In Internet there is a PC with a video streaming server. It allows us to measure the different features of each device. All devices start to watch a video from the server at the same time and meanwhile Wireshark [18] is used to sniff the network traffic for monitoring purposes.

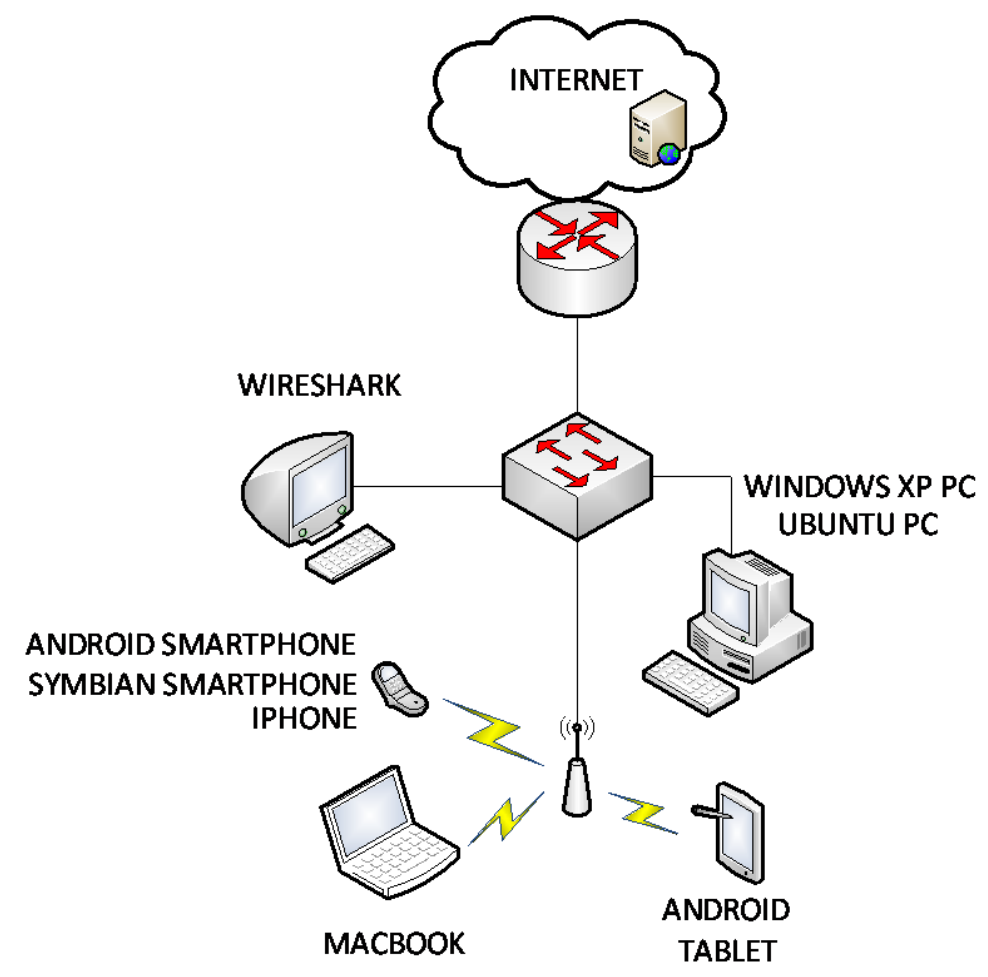

Figure 1. Network Topology

\subsection{QoS Parameters Experiment}

This experiment let us measure the round trip time, delay and jitter of mobile and fixed devices. Fig. 2, 4 and 6 show these parameters for the group of mobile devices and Fig. 3, 5 and 7 for the fixed devices.

As expected, these figures show that the time required for mobile devices to transmit and receive packets from a network is higher than for fixed devices because their packets have to cross the wireless network.

The round trip time is the time required to transmit one TCP packet from a source device to a specific destination plus the time required for the acknowledgment to get back to the source from the destination. Fig. 2 and 3 show this time for mobile and fixed devices respectively. As we can see, the round trip time for fixed devices is almost zero, lower than 0.05 seconds, apart from some specific peaks; in contrast, this time has an average of 0.2 seconds for mobile devices. 


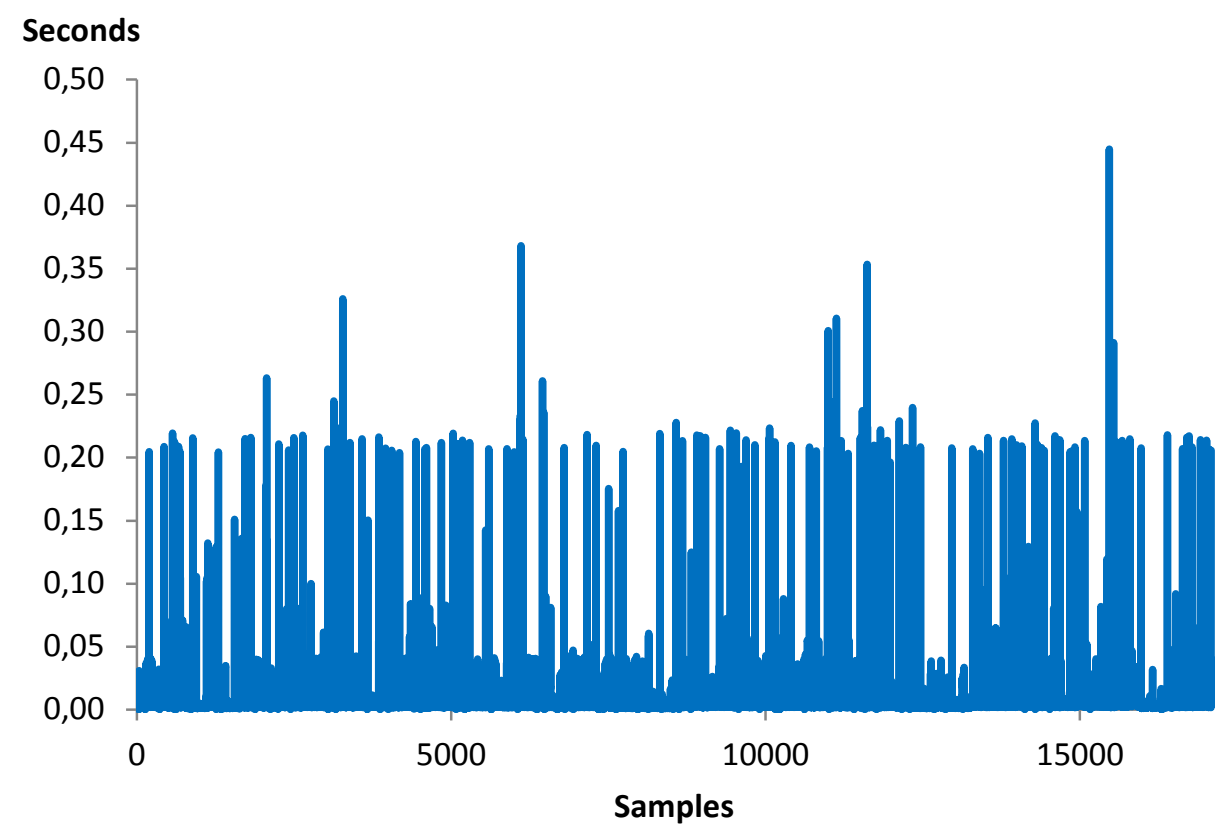

Figure 2. Round Trip Time in Mobile Devices

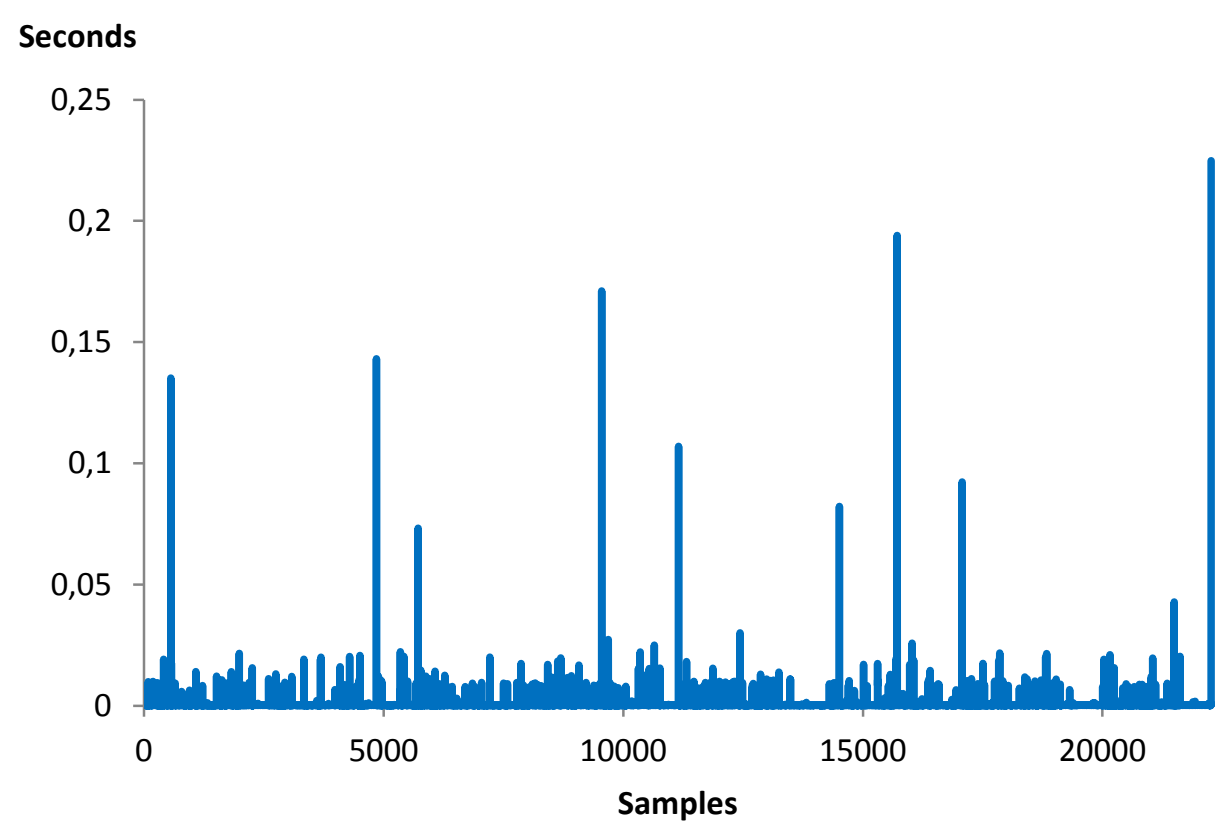

Figure 3. Round Trip Time in Fixed Devices

The same happens when we measure the delay, which is the time required for a TCP message to travel from a source to a destination. Fig. 4 and 5 show that the delay for mobile devices has an average value of 0.10 seconds and it is lower than for fixed devices, which is 0.02 seconds. 


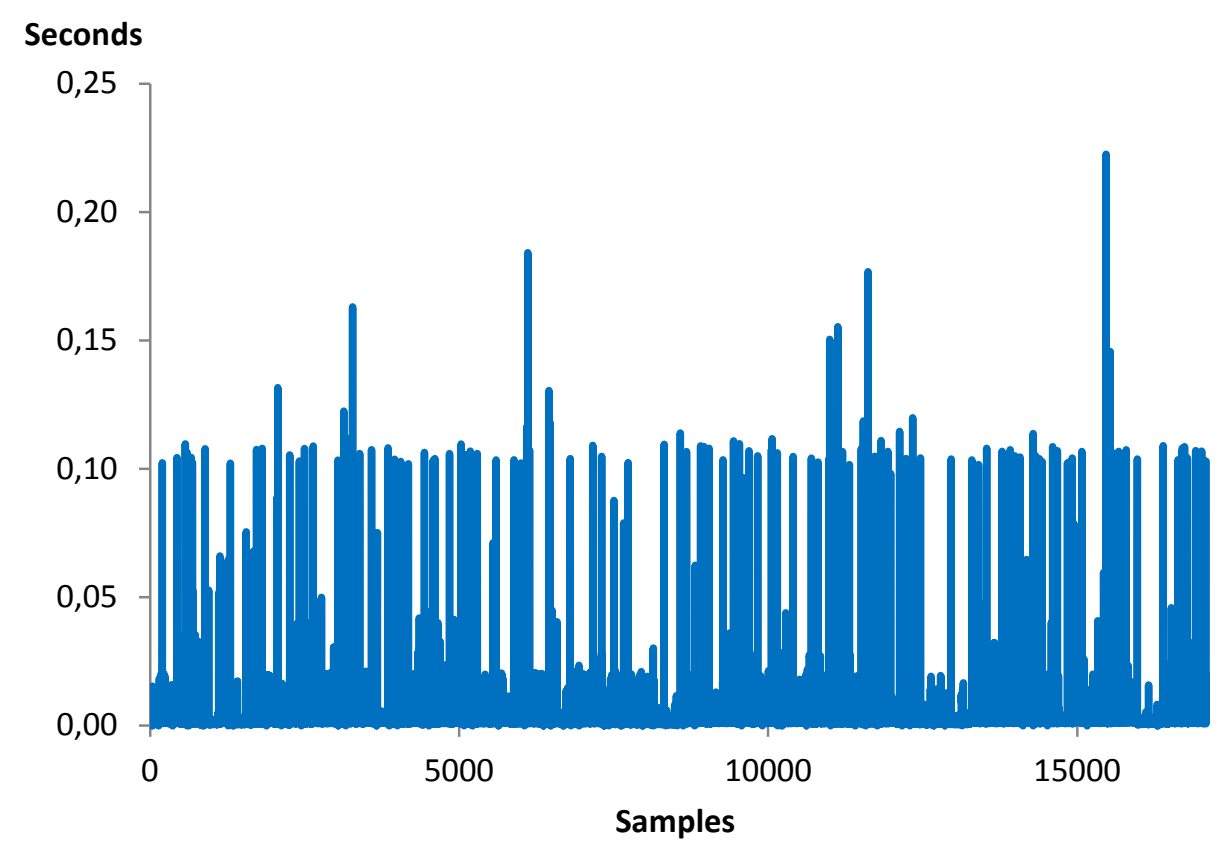

Figure 4. Delay in Mobile Devices

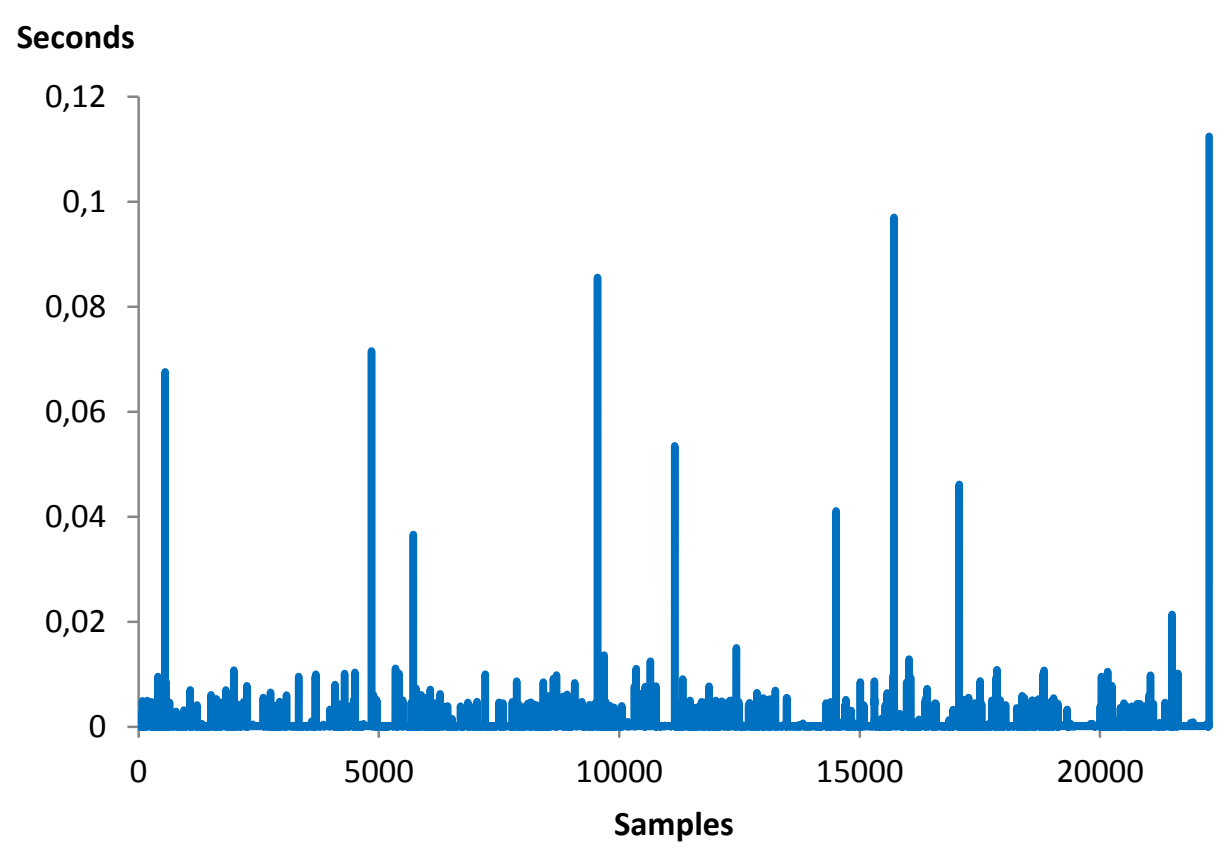

Figure 5. Delay in Fixed Devices

When we measured the jitter, which is the difference between the delay of two consecutive packets, we also obtained different values. Fig. 6 shows that these values are 0.10 seconds in the most of cases for mobile devices and, in Fig. 7, it is shown that these values are around zero for fixed devices. 


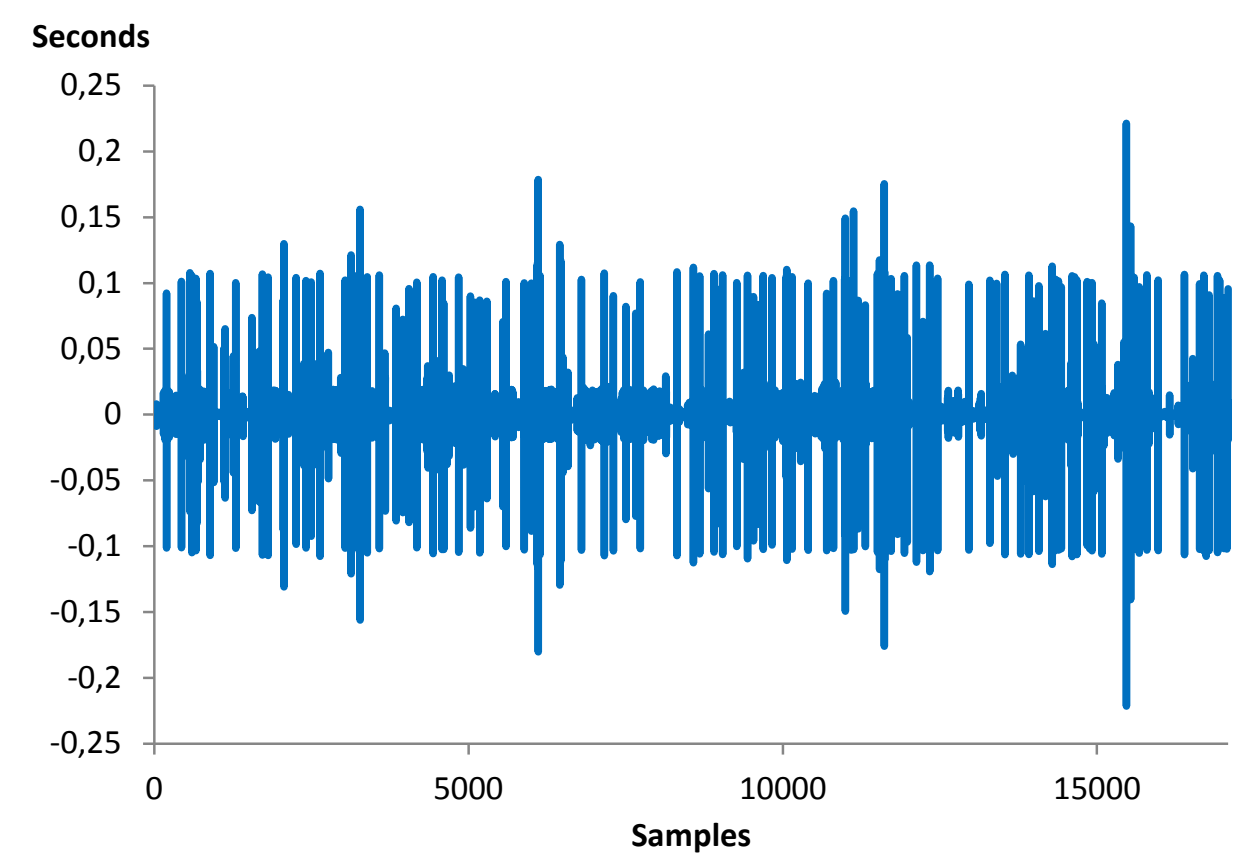

Figure 6. Jitter in Mobile Devices

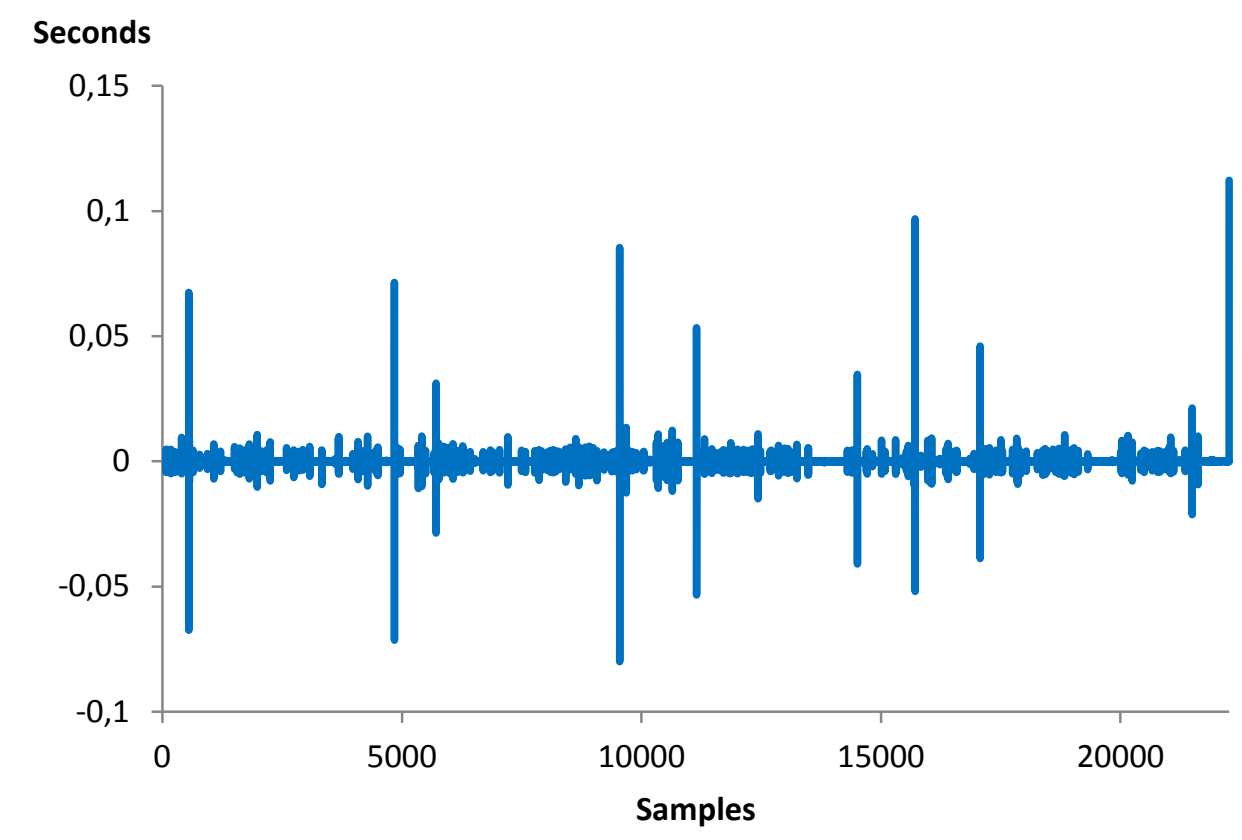

Figure 7. Jitter in Fixed Devices

As expected, the throughput needed is also different for mobile and fixed devices. But, as we can see in Fig. 8 and 9, this difference is not so significant in this case.

Fig. 8 and 9 show the overall packets and bytes transmitted by mobile and fixed devices while they are watching the video received from the video server. As we can see the throughput is a little bit higher for fixed devices than for mobile devices at the beginning of the transmission. It makes sense because the bandwidth is usually greater in fixed devices. However, it decreases slightly at the end of the transmission in fixed devices and it remains similar during all the transmission in mobile devices. 


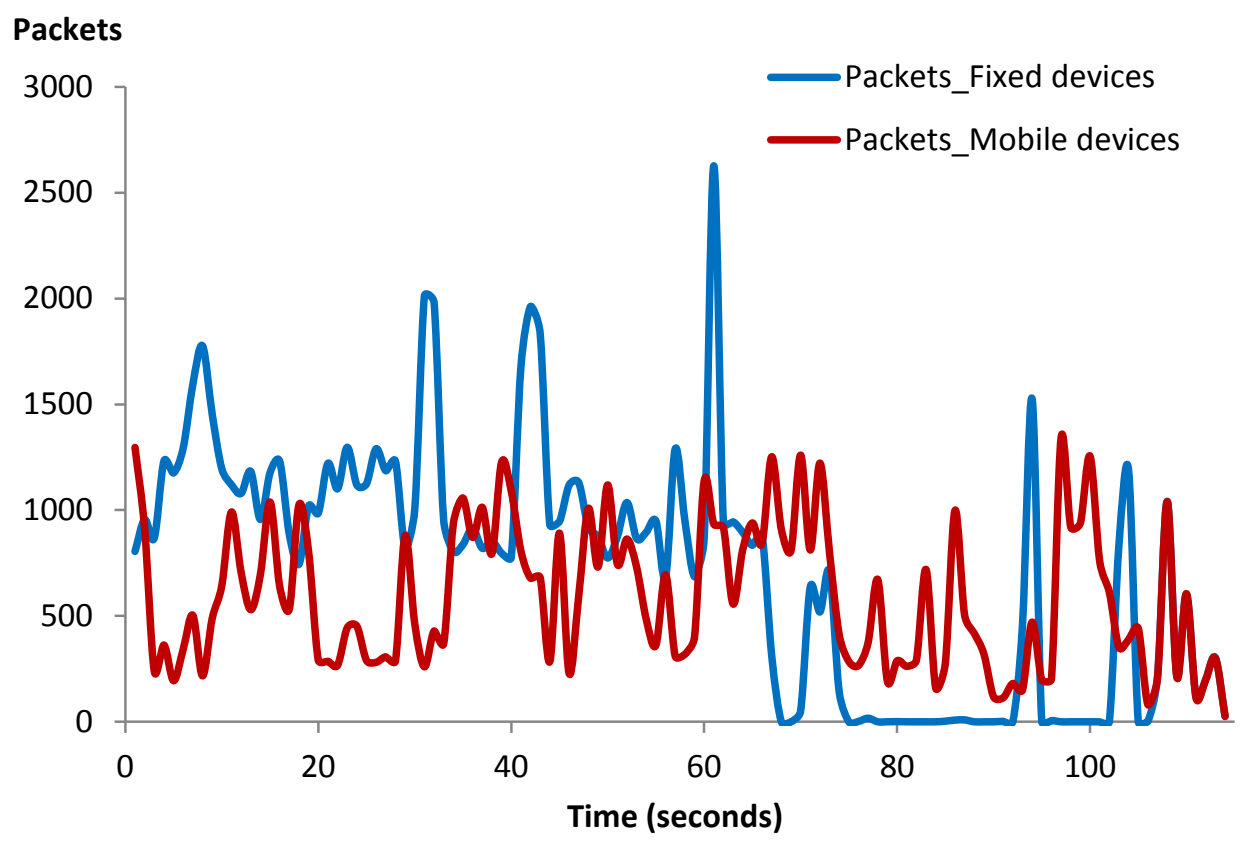

Figure 8. Consumed throughput in packets per second

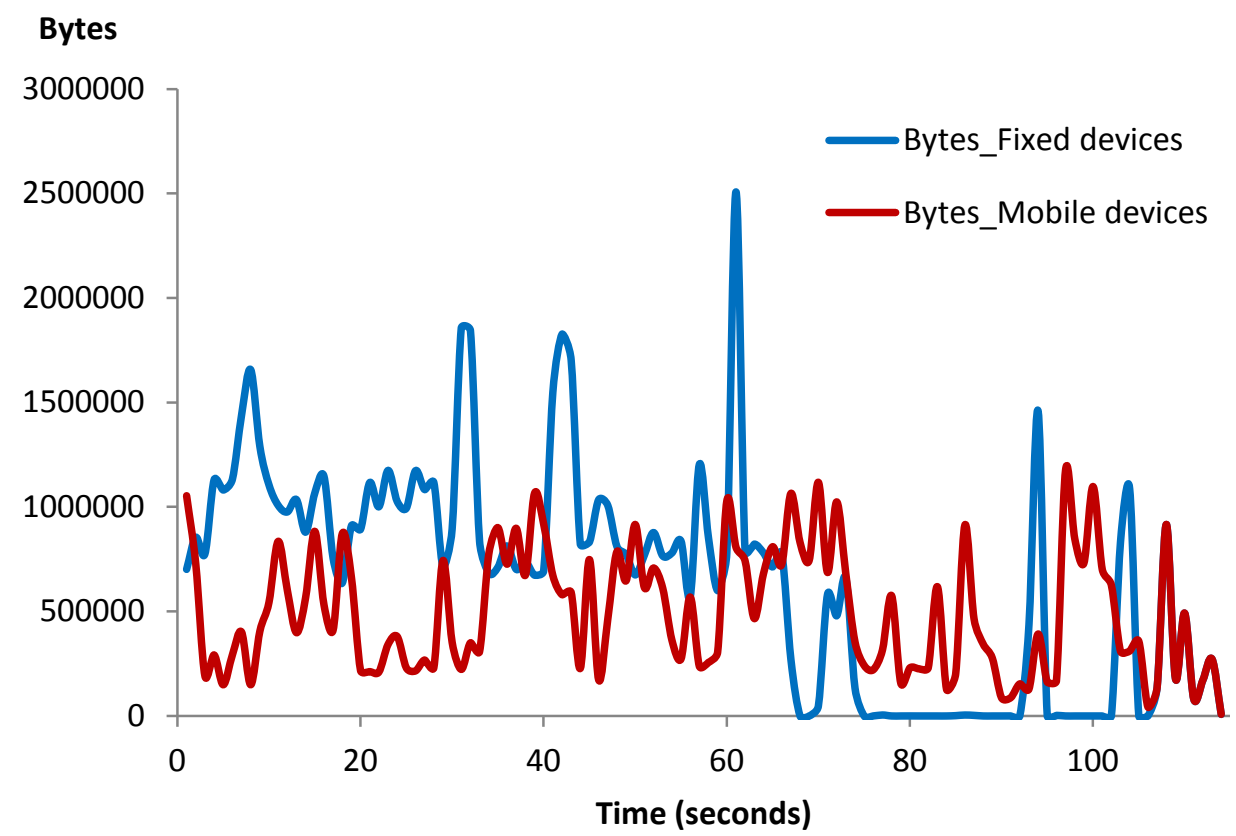

Figure 9. Consumed throughput in bytes per second

\subsection{Application Layer Experiment}

The goal of this experiment is to find differences at the application layer. We have found that for the HTTP protocol, the user-agent field allows us to identify both the operating system and the device. Moreover, we have found differences in the TTL, WS and OBS variables. The presence of the DF bit determines that these messages are the first ones of the communication. 
The following list shows the information obtained in the field user-agent for all tested devices:

- SonyEricsson ST 25i: Mozilla/5.0 (Linux; U; Android 2.3.7; es-es; SonyEricssonST25i Build/6.0.B.1.564)

- $\quad$ iphone 5: com.google.ios.youtube/1.2.1.5201 (iPhone5,2; U; CPU iPhone OS 6_1_2 like Mac OS X; es_ES)

- Nokia C7: Mozilla/5.0 (Symbian/3; Series60/5.3 NokiaC7-00/111.030.0609; Profile/MIDP-2.1 Configuration/CLDC-1.1)

- Nokia C6: Opera/9.80 (S60; SymbOS; Opera Mobi/SYB-1204232254; U; es-ES)

- LG-P700: Mozilla/5.0 (Linux; U; Android 4.0.3; es-es; LG-P700 Build/IML74K)

- Macbook Pro: Mozilla/5.0 (Macintosh; Intel Mac OS X 10_6_8)

- Acer A500: Mozilla/5.0 (Linux; U; Android 3.2.1; es-es; A500 Build/HTK55D)

- PC (Windows XP): Mozilla/5.0 (Windows NT 5.1)

- PC (Linux Ubuntu 10.04): Mozilla/5.0 (X11; Linux x86_64)

Moreover, in our laboratory, after several experiments, we have confirmed that the three variables used by R. Beverly in [12] to classify operating system have slight differences depending on the device and the operating system that is being connected to Internet. Table 1 shows the obtained values for these variables.

Table 1. TTL, Window Size and Options Block Size Comparison.

\begin{tabular}{|l|l|l|l|}
\hline \multicolumn{1}{|c|}{ Device (Operating System) } & \multicolumn{1}{c|}{ TTL } & \multicolumn{1}{c|}{ WS } & \multicolumn{1}{c|}{ OBS } \\
\hline \hline Smartphone (Nokia C6 Symbian S60) & 69 & 64,980 & 12 \\
\hline Iphone (iOS 6) & 64 & 8,192 & 12 \\
\hline Smartphone (Nokia C7 Symbian OS 9.5 Symbian^3) & 69 & 12,227 & 12 \\
\hline Smartphone (LG-p700 Android 4.0) & 64 & 229 & 12 \\
\hline Smartphone (SonyEricsson Android 2.3) & 64 & 2,920 & 12 \\
\hline Tablet (Acer A500 Android 3.2) & 64 & 1,004 & 12 \\
\hline PC (Windows XP) & 128 & 65,535 & $0 / 8$ \\
\hline PC (Linux Ubuntu 10.04) & 64 & 501 & 12 \\
\hline MacBook Pro (OSX 10.6.8) & 64 & 524,280 & 12 \\
\hline
\end{tabular}

\subsection{Transport Layer Experiment}

The aim of this second experiment is to confirm that the values of TTL, WS and OBS are independent of the used protocol. Using the same configuration shown in Fig. 1 we have taken several messages from the nine devices using different protocols. In order to do this experiment we have used Skype, Google Talk and Youtube.

We have observed the same values for TTL, WS and OBS (TTL=63, WS=524280 and OBS=12). They are the same values than the ones used by Mac OSX 10.6 for HTTP protocol (see Table 1). 
In one of our experiments, we saw a TTL value of 68 . We know from Table 1 that TTL $=$ 69 represents Nokia smartphones. So, it is not possible to know the type of device only using the TTL value, but it is possible comparing this value with the value in others messages in the network. For example, in our experiments we were able to identify Nokia devices when there are more messages from others devices (including fixed devices), because they have higher TTL values.

Next, we performed some experiments in order to detect which device is used to watch a video from Youtube. We observed that Youtube uses the HTTP protocol, for this reason the values that we observe are the same than in Table 1.

We also obtained $\mathrm{OBS}=0$, which indicates the absence of the options block. The values of these variables are comparable with the values of a computer with Windows XP for HTTP protocol (shown in Table 1).

\subsection{Data Flow Experiment}

Last experiments permit to identify the device only on the first message of the communications. In this experiment we wanted to identify the device responsible of the data flow in two senses. In order to perform it, we used the identification of the device to determine the class of the device (Fixed device / Mobile device) and the sockets information in the first message to make a 5-tuple (IP direction / port source, IP direction / port destination, class) and we have stored this information on a connection tracking table.

\section{Device Recognition System}

Bearing in mind all performed experiments, we have developed a system that lets us know which type of device is the origin of the traffic.

In order to achieve our goal we have designed and developed an intelligent system that is able to classify and measure the TCP/IP messages that come from different mobile devices and computers, by using the available information in the application and transport layers [19]. In this intelligent classifier, we use a neural network in order to take the most accurate results.

The block diagram structure is shown in Fig. 10. As we can see, our system is composed by seven blocks:

- libPCAP: Monitor the network traffic and capture packets.

- Packet analyzer: Extracts the main features shown on Table 2 of the network packet.

- Neural Network: Estimates the device class for the packets containing don't fragment bit.

- Connection Tracking: Finite state machine with two states (Computer and Mobile device) that uses the information on the connection tracking \& class table as state transition rules. This block sends the packet class to statistics block. 
- Connection Tracking \& Class Table: Table where the sockets communication information and class is stored in a 5-tuple.

- Statistics: Count the amount of packets of each class in the net from the start of the system.

- Output: Shows statistics information to the user.

In other words, by using libPCAP [20], our system can read network packets. Then, these packets are sent to the analyzer block. Next the analyzer block extracts the main features (see Table 2) and builds features vectors. These features vectors are send to the neural network block and to the connection-tracking block. Our neural network is able to recognize which device is the origin of the communication by the first packet of this communication, but it is not able to do it for the others packets. For this reason, it is necessary to work also with a flow TCP basis [21]. The connection-tracking block is a finite state machine with two possible states: computer and mobile device, which uses the information on the connection tracking \& class table as state transition rules. The flow information is represented by a 5-Tuple (IP address / port source, IP address / port destination, state), and the current state is saved on the connection-tracking table. The statistics block is responsible for counting the packets received from each class, and prepares the system output, which is the percentage of each class.

The main features of the network packets needed to recognize the device class and to monitor the traffic flow are shown on Table 2. The automatic labeling method used to label the features vectors for the training of the neural network is shown in Fig. 10. This block diagram is composed by:

- libCAP.

- Packet analyzer.

- Device Table.

- Output: Builds an arff file with the information on Device table to train the neural network using Weka [22].

During the packet-labeling period, the set of packets captured by libCAP are examined by the packet analyzer to build the set of main features vectors with the values of the TTL, OBS and WS of the packets with the DF. Next, it labels features vectors according to the value of the user-agent. This field has two classes: Computer and Mobile device.

All these vectors were collected in the device table and used to make the arff file to train the neural network with Weka. 


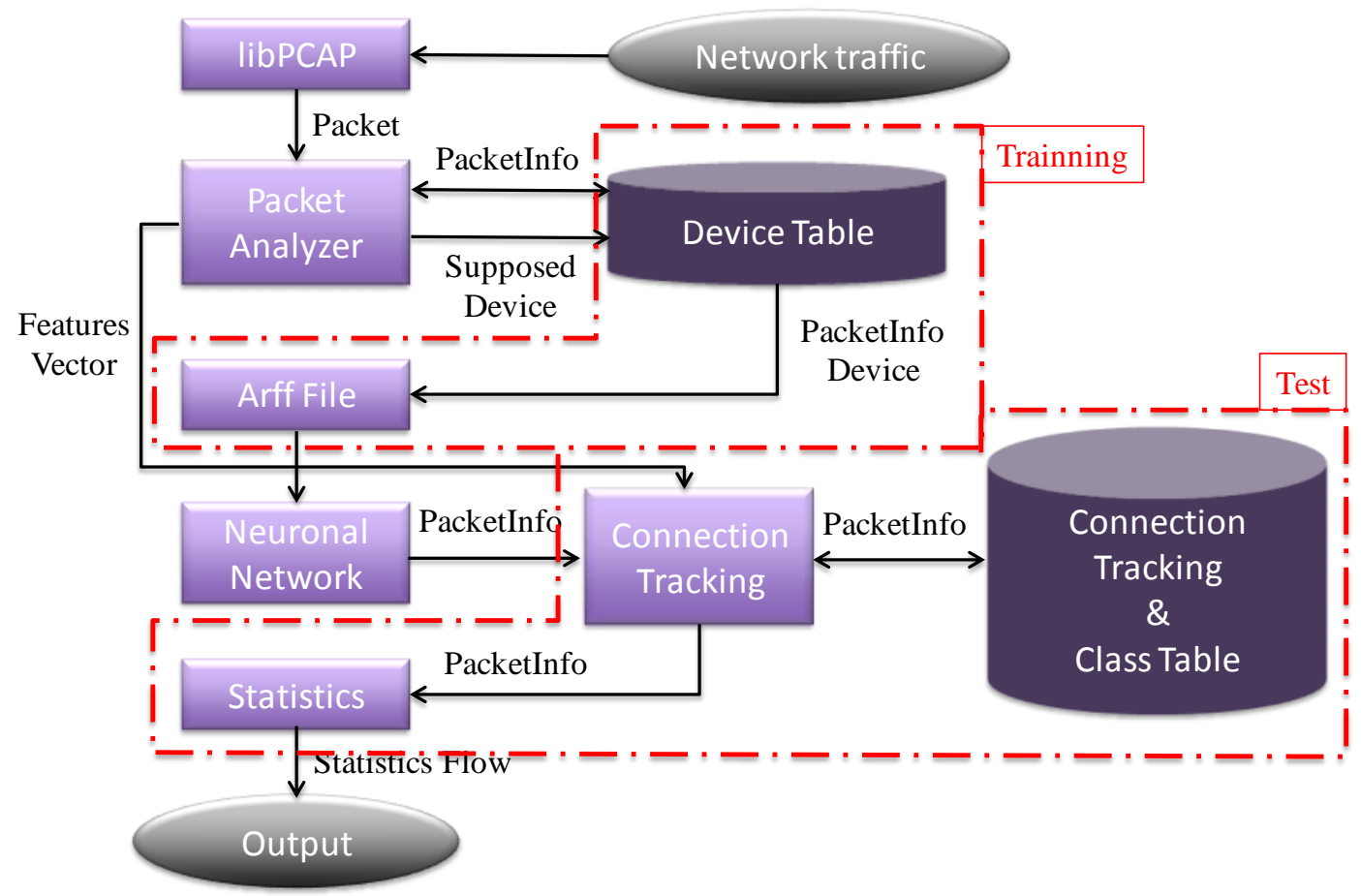

Figure 10. Block diagram of statistics computation.

Table 2. Main features of a network packet.

\begin{tabular}{|l|l|}
\hline \multicolumn{1}{|c|}{ Name } & Abbreviation \\
\hline \hline Protocol & P \\
\hline Time to Live & TTL \\
\hline Options Block Size & OBS \\
\hline Don't fragment bit & DF \\
\hline Window size & WS \\
\hline IP address source & IPS \\
\hline Port source & PS \\
\hline IP address destination & IPD \\
\hline Port destination & PD \\
\hline
\end{tabular}

\section{Device Recognition System Experiments}

By doing some experiments, we have developed a system which allows us to identify which type of device is the source of the traffic.

\subsection{Analytical model.}

The neural network used in our system is a multilayer perceptron [23]. In order to find a network that maximizes the classification results, we performed a training phase using a set of training samples is given with their corresponding class labels. It is represented by : 


$$
A=\left\{\left(\vec{x}_{i}, y\right) \mid \vec{x}_{i} \in \mathbb{R}^{d}, 1 \leq y \leq M \text { for } 1 \leq i \leq n\right\}
$$

Where,

$\vec{x}_{i}$ are the training samples that will be used as inputs in our network

$y_{i}$ are the class labels

$M$ are the family class discriminant functions

The class labels are defined by:

$$
y_{i}=\left\{\begin{array}{l}
1 \quad \text { if } \quad \vec{x}_{i} \text { is of class } M \\
0 \quad \text { if } \vec{x}_{i} \text { is not of class } M
\end{array}\right.
$$

Our aim is to find a probabilistic approach, where given an input sample, the network will provide us the corresponding class, fulfilling $\operatorname{Pr}\left(y \mid \vec{x}_{i}\right)$.

The activation function used in our neural network to carry out the training phase is a linear function. Therefore, it fulfills that:

$$
f\left(z_{j}\right)=z_{j}
$$

The topology of our neural network is shown in Fig. 12. This is a multilayer perceptron with three layers, three nodes in the input layer (TTL, WS and OBS), ten nodes in the hidden layer and two nodes on the output layer (fixed and Mobile).

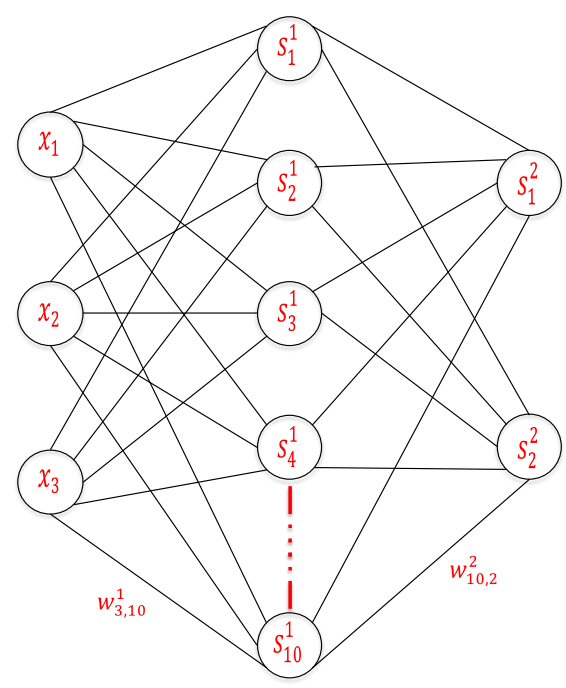

Figure 12. Neural network topology 
This topology is defined by $T=(V, C)$, where,

$V_{0}$ are the set of input nodes,

$V$ are the rest of the nodes defined by discriminant functions,

$C$ are the connectors

$\vec{w}$ are the weights of each connection

The algorithm procedure is shown in Fig. 13. For each input pattern, the system performs a forward sweep to find the actual output. Then, it estimates the network error. If it is higher than a threshold then it executes the backpropagation to determine weight changes and update them.

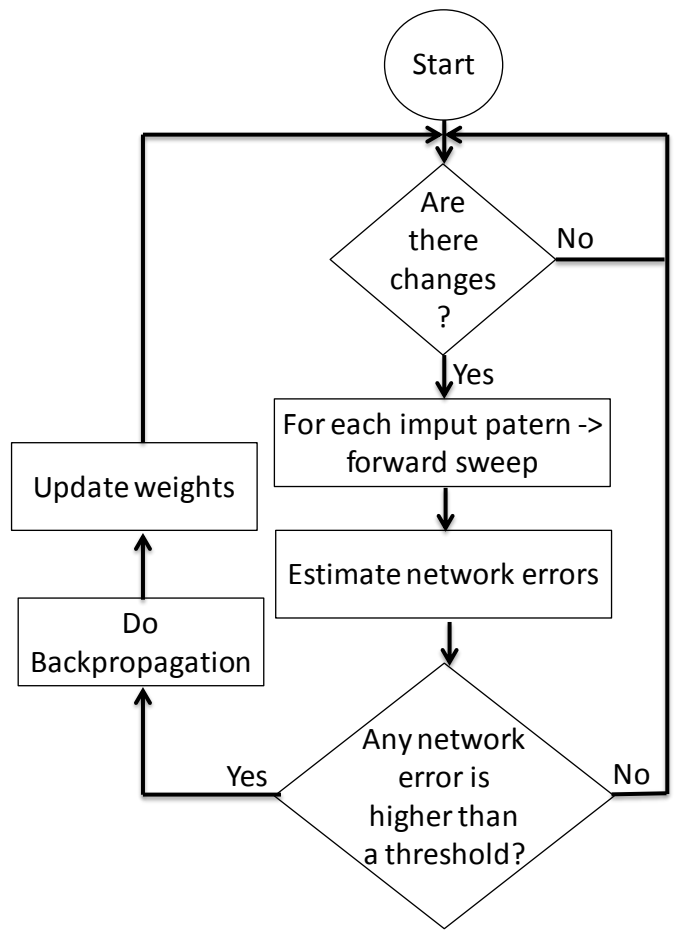

Figure 13. Algorithm procedure.

The multilayer perceptron is defined by a combination of linear discriminant functions grouped into two layers as it is given in:

$$
s_{y}^{2}(\vec{x})=f\left(\sum_{j=0}^{N_{1}} w_{y j}^{2} s_{j}^{1}(\vec{x})\right)=f\left(\sum_{j=0}^{N_{1}} w_{y j}^{2} f\left(\sum_{j^{\prime}=0}^{N_{0}} w_{j j^{\prime}}^{1} x_{j^{\prime}}\right)\right)
$$

Because the activation functions are linear, equation (4) can be expressed as:

$$
s_{y}^{2}(\vec{x})=\sum_{j^{\prime}=0}^{N_{0}}\left(\sum_{j=0}^{N_{1}} w_{y j}^{2} w_{j j^{\prime}}^{1}\right) x_{j^{\prime}}=\sum_{j^{\prime}=0}^{N_{0}} w_{y j^{\prime}} x_{j^{\prime}}
$$


The neurons of the hidden layer are between $1 \leq j \leq N_{1}$, where $N_{1}=10$. The neurons of the input layer are between $1 \leq j^{\prime} \leq N_{0}$, where $N_{0}=3$, and the ones of the output layer are between $1 \leq y \leq N_{2}$, where $N_{2}=2$.

Finally, we define the learning algorithm which minimizes the classification error. Backpropagation has been the used algorithm. Therefore, given the topology $T=(V, C)$ of the multilayer perceptron and the set $A=\left\{\left(\vec{x}_{1}, y_{1}\right), \ldots,\left(\vec{x}_{n}, y_{n}\right)\right\}$, with $\vec{x}_{p} \in \mathbb{R}^{N_{0}}, t_{p} \in \mathbb{R}^{N_{2}}$, the objective is to find $\vec{w} \in \mathbb{R}^{|C|}$ which minimizes the mean square error for $\mathrm{n}$ training samples.

So, given the error in:

$$
E_{A}(\vec{w})=\frac{1}{2 n} \sum_{p=1}^{n} \sum_{y=1}^{N_{2}}\left(t_{p, y}-s_{y}^{2}\left(\vec{x}_{p}\right)\right)^{2}
$$

we minimize it by the descending gradient method:

$$
\Delta w_{i j}^{k}=-\rho \frac{\partial E_{A}(\vec{w})}{\partial w_{i j}^{k}}
$$

In this algorithm, the update of the weights in both hidden and output layer, for all training samples $(n=40920)$, each iteration is defined by the sigmoidal function-common in Multi Layer Perception (the sigmoidal function gives a value in range of 0 to 1 ):

1) In the output layer, for $1 \leq i \leq N_{2}, 1 \leq j \leq N_{1}$ we have:

$$
\begin{aligned}
& \Delta w_{i j}^{2}=\rho \sum_{p=1}^{n} \delta_{i}^{2}\left(\vec{x}_{p}\right) s_{j}^{1}\left(\vec{x}_{p}\right) \\
& \delta_{i}^{2}\left(\vec{x}_{p}\right)=\left(t_{i p}-s_{i}^{2}\left(\vec{x}_{p}\right)\right) f^{\prime}\left(e_{i}^{2}\left(\vec{x}_{p}\right)\right)
\end{aligned}
$$

2) In the hidden layer, for $1 \leq i \leq N_{1}, 0 \leq j \leq N_{0}$ we have:

$$
\begin{aligned}
& \Delta w_{i j}^{1}=\rho \sum_{p=1}^{n} \delta_{i}^{1}\left(\vec{x}_{p}\right) x_{p j} \\
& \delta_{i}^{1}\left(\vec{x}_{p}\right)=\left(\sum_{r} \delta_{r}^{2}\left(\vec{x}_{p}\right) w_{r i}^{2}\right) f^{\prime}\left(e_{i}^{1}\left(\vec{x}_{p}\right)\right)
\end{aligned}
$$

Ideally, when it finds a global minimum, the function converges. It happens when equation (12) happens.

$$
\operatorname{Pr}\left(y \mid \vec{x}_{i}\right)=s_{y}^{2}(\vec{x})
$$

So, if we arrive to this situation, the network will be overtraining. Therefore, in order to avoid it, we have selected two criteria to stop the system, one based on the number of epochs 
and another focused on the validation error.

To sum up, given an input conditions such as network topology, training set, learning factor and convergence conditions and after performing the backpropagation algorithm, we obtain the weights of the connections which minimizes the mean square error .

Because we have a balanced data model, we can take as a classification measurement the accuracy defined in [24] by H. He and E. A. Garcia. However we will also take another error value, which is the precision.

\subsection{Test Bench}

The process is divided into two parts. On the one hand the automatic labeling of samples to perform the neural network training and on other hand the statistical calculation test.

In order to test the proposed system, we used the devices shown in Table 1 to generate network traffic. After the automatic filtering and labeling we had a set of 14,429 samples of packets from mobile devices, and 36,721 samples of packets from computers. We split it in $80 \%$ for training set and $20 \%$ for validation set. Table 3 contains the number of samples for training and validation sets.

Table 3. Number of samples for training and validation sets.

\begin{tabular}{|l|c|c|}
\hline \multicolumn{1}{|c|}{ Class } & Training set & Validation set \\
\hline \hline Mobile device & 11,543 & 2,886 \\
\hline Fixed device & 29,377 & 7,344 \\
\hline
\end{tabular}

We trained this multilayer perceptron using 10-folds cross validation. Based on the confusion matrix shown in Table 4, we obtain the training results shown in equation (13) and (14).

Table 4. General confusion matrix

\begin{tabular}{|l|l|l|}
\hline Prediction/real & Positive & Negative \\
\hline \hline Positive & VP & FP \\
\hline Negative & FN & VN \\
\hline
\end{tabular}

Precission $=\frac{V P}{V P+F P}=86.2 \%$

$$
\text { Accuracy }=\frac{V P+V N}{V P+F P+V N+F N}=86.4 \%
$$

Finally, we have also taken into account the error obtained on the area under the ROC (Relative Operating Characteristic) curve which has resulted in $91.3 \%$. The ROC curve 
analysis provides tools for selecting optimal models and possibly discards suboptimal models irrespective of the cost of the distribution of the two classes is decided on. A random method describes a ROC curve horizontally through the diagonal, with a value of $50 \%$. The classifiers at least should make the classification better than this, so far their performance is related to the area under the ROC curve, the larger this area is the better the performance of the classifier is.

Once the neural network was trained, we generated this model and incorporated it to the system in order to test the whole system with the complete traffic trace generated before. This trace was composed by 47,642 packets from mobile devices and 75,307 from fixed devices. Thus, the expected values are $38.75 \%$ for mobile devices and $61.25 \%$ for fixed devices.

As we can see in Fig. 14, the observed values on the system output were $33.90 \%$ of packets as traffic from mobile devices and the $66.10 \%$ of packets as traffic from fixed devices (computers), comparing the expected values with the observed we found a $4.85 \%$ of error in global classification.

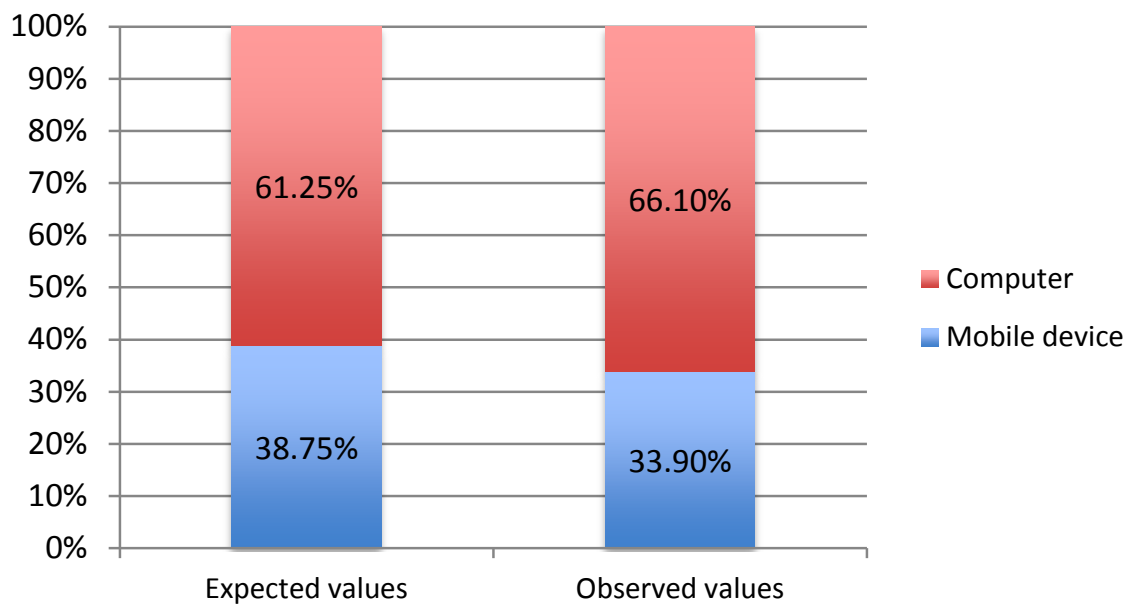

Figure 14. Expected and observed values on the output.

In Table 5 the confusion matrix is shown with numerical values. It let us know the classifiers performance in detail. We see that 12,816 samples of mobile devices where classified as fixed devices. It means $26.90 \%$ of error in the mobile devices class. For the fixed device class we see that there were 6,858 samples classified as mobile devices. It means $9.11 \%$ of error in fixed device class.

Table 5. Confusion matrix.

\begin{tabular}{|l|l|l|l|}
\hline & & & \multicolumn{1}{|c|}{ Predicted Class } \\
\hline \hline & & Mobile Device & Fixed Device \\
\hline & Mobile device & 34,826 & 12,816 \\
\hline Actual Class & Fixed Device & 6,858 & 68,449 \\
\hline
\end{tabular}




\section{Conclusion}

In this paper we have presented an intelligent system to detect the type of the device generating Internet traffic in the data network of the Internet Service Provider. The neural network used in our system is a multilayer perceptron. We have concluded that it is possible to recognize the type of devices behind of a router using Network Address Translation by monitoring small variations in certain variables. These variations generate patterns based on the operating system, which can be easily recognized by an intelligent system. The success of our implemented system demonstrates it.

In future works we will include in our system a reinforcement-learning block. This new block will be able to learn by the use, and will reduce the error in classification, especially in mobile devices class.

\section{References}

[1] M. Zhang, W. John, K. Claffy, and N. Brownlee, "State of the art in traffic classification: A research review," Passive and Acctive Measurement Conference, Seoul, South Korea, April $1-3,2009$.

[2] T.T.T. Nguyen and G. Armitage, "A survey of techniques for internet traffic classification using machine learning," IEEE Communications Surveys \& Tutorials, vol. 10, no. 4, pp. 56-76, 2008. http://dx.doi.org/10.1109/SURV.2008.080406

[3] D. Zuev and A. W. Moore, "Traffic Classification Using a Statistical Approach," 6th international conference on Passive and Active Network Measurement (PAM'05), Boston, MA, USA, March 31-April 1, 2005. http://dx.doi.org/10.1007/978-3-540-31966-5_25

[4] M. Crotti, M. Dusi, F. Gringoli, and L. Salgarelli, "Traffic Classification through Simple Statistical Fingerprinting," ACM SIGCOMM Computer Communication Review. vol. 37, Issue 1, pp. 5-16, January 2007. http://dx.doi.org/10.1145/1198255.1198257

[5] D. Moore, K. Keys, R. Koga, E. Lagache, and K. C. Claffy, "The CoralReef Software Suite as a Tool for System and Network Administrators," 15th USENIX conference on Systems Administration (LISA'01), San Diego, CA, USA, December 2001.

[6] J. Erman, M. Arlitt, and A. Mahanti, "Traffic Classification Using Clustering Algorithms," Special Interest Group on Data Communication Conference (SIGCOMM'06), Pisa, Italy, September 11-15, 2006. http://dx.doi.org/10.1145/1162678.1162679

[7] A. W. Moore and D. Zuev, "Internet Traffc Classification Using Bayesian Analysis Techniques," the 2005 ACM SIGMETRICS international conference on Measurement and modeling of computer systems (SIGMETRICS'05), Banff, AB, Canada, June 06 - 10, 2005. http://dx.doi.org/10.1145/1071690.1064220

[8] T. Karagiannis, A. Broido, N. Brownlee, K.C. Claffy, and M. Faloutsos, "Is P2P dying or just hiding? [P2P traffic measurement]," IEEE Global Telecommunications Conference 
(GLOBECOM '04), Dallas, Texas, November 29-December 3. http://dx.doi.org/10.1109/GLOCOM.2004.1378239

[9] T. Karagiannis, K. Papagiannaki, and M. Faloutsos, "BLINC: Multilevel Traffic Classification in the Dark," Special Interest Group on Data Communication Conference (SIGCOMM'05), Philadelphia, Pennsylvania, USA, August 22-26, 2005. http://dx.doi.org/10.1145/1080091.1080119

[10] Yahoo! and The Nielsen Company, "The Mobile Shopping Framework Study: The Role of Mobile Devices in the Shopping Process," White paper, January 2011. http://advertising.yahoo.com/article/the-role-of-mobile-devices-in-shopping-process.html (Last Access, June 2013)

[11] Douglas E. Comer and John C. Lin, "Probing TCP implementations", USENIX Summer 1994 Technical Conference, Boston, USA, June 6-10, 1994.

[12]R. Beverly, "A robust classifier for passive TCP/IP fingerprinting," Passive and Active Network Measurement, Lecture Notes on Computer Science, vol. 3015, pp. 158-167, 2004. http://dx.doi.org/10.1007/978-3-540-24668-8_16

[13] Emilio Granell, Diana Bri, Jesus Tomas and Jaime Lloret, Smart Devices Fingerprint Detection, IEEE Global Communications Conference (IEEE Globecom 2012), Anaheim, $\begin{array}{llllll}\text { California, } & \text { USA, } & \text { December } & 3 & - & 7,\end{array}$ http://dx.doi.org/10.1109/GLOCOMW.2012.6477587

[14]E. Hjelmvik and W. John, "Statistical protocol identification with spid: Preliminary results," Swedish National Computer Networking Workshop (SNCNW'09), Uppsala, Sweden, May 4-5, 2009

[15] Saumil Shah, An Introduction to HTTP fingerprinting, Net-Square Solutions, 19th May, 2004. Available at: http://www.net-square.com/httprint_paper.html (Last Access, June 2013)

[16]P. Eckersley, "How unique is your web browser?," 10th international conference on Privacy enhancing technologies (PETS'10), Berlin, Germany, July 21-23, 2010

[17]J. Oshio, S. Ata, and I. Oka, "Identification of different TCP versions based on cluster analysis," IEEE 18th International Conference on Computer Communications and Networks (ICCCN 2009), San Francisco, CA, August 3-6, 2009. http://dx.doi.org/10.1109/ICCCN.2009.5235248

[18] Wireshark (2012) http://www.wireshark.org/ (Last Access, June 2013)

[19]M. Crotti, M. Dusi, A. Este, F. Gringoli, and L. Salgarelli, "Application Protocol Fingerprinting for Traffic Classification," GTTI Riunione Annuale 2007, Roma, Italia, June 18-20, 2007.

[20] TCPDUMP \& libPCAP (2010) http://www.tcpdump.org/ (Last Access, June 2013)

[21]T.A. Beardsley and J. Qian, "The TCP Split Handshake: Practical Effects on Modern Network Equipment," Network Protocols and Algorithms, vol. 2, no. 1, pp. 197-217, 2010. 
http://dx.doi.org/10.5296/npa.v2i1.285

[22] Weka. Available at: http://www.cs.waikato.ac.nz/ml/weka/ (Last Access, June 2013)

[23] Christopher M. Bishop, Neural Networks for Pattern Recognition, Oxford University Press (1995). ISBN 0-19-853864-2 (Paperback).

[24] Haibo He and Edwardo A. Garcia, Learning from Imbalanced Data, Journal IEEE Transactions on Knowledge and Data Engineering, Volume 21 Issue 9, September 2009. Pp. 1263-1284. http://dx.doi.org/10.1109/TKDE.2008.239

\section{Copyright Disclaimer}

Copyright reserved by the author(s).

This article is an open-access article distributed under the terms and conditions of the Creative Commons Attribution license (http://creativecommons.org/licenses/by/3.0/). 